\title{
The Journal of Clinical Investigation in the time of COVID-19
}

\author{
In this editorial, we describe the experience of the $J C l$ editors during the \\ COVID-19 pandemic. Our goal is to share how we operated during the \\ pandemic, recount how the $\mathrm{JCl}$ contributed to the response, highlight some \\ of the major papers we published on SARS-CoV-2 and COVID-19, and impart \\ our insights in the hope that these are helpful to journal editors that may \\ need to deal with similar types of crises in the future.
}

In the early days of March 2020, most people in the United States were still going about life as usual. Despite everything seeming outwardly normal, there was great concern that COVID-19, which was then ravaging China and Europe, would spread to the United States. The first COVID-19 case in the United States was confirmed on January 21, 2020 (1), but fewer than 100 confirmed cases had been reported by March 1, 2020. By the end of the month, much of the United States was in lockdown, with severe disruptions to work, travel, and the rhythms of everyday life, and COVID-19 cases were rapidly increasing in the Northeast. The situation in Northeastern cities such as New York reached crisis proportions as hospitals filled with contagious COVID-19 patients, nonspecialist providers were deployed to care for an infectious disease, and protective equipment was in short supply. The pandemic still rages and the cost in human life in the United States alone has been devastating, with more than 750,000 deaths, a number that exceeds the toll of the 1918 influenza pandemic.

\section{Pandemic operation}

The JCI is unusual among scholarly journals in that it is led by an Editorial Board composed primarily of physician-scientists based at one institution, with the Editor in Chief chosen for a five-year term by the American Society of Clinical Investigation in a competitive process (2). The JCI Editorial Board was primarily based at Johns Hopkins University starting in 2017, where it met every Tuesday from 9 to $11 \mathrm{am}$ to discuss the disposition of manuscripts that had been reviewed. The last in-person JCI board meeting occurred on March 10, 2020. Afterward, the board met virtually, using the Zoom platform to hold our meetings (Figure 1). The first online meeting was on March 17, 2020, and the board continues to meet virtually at this time. The transition from in-person to virtual meetings was smooth, with members rapidly adapting to the new format. Since our editors had transitioned to working at home and work-related travel had ceased, the virtual board meetings were very well attended. For our board members based at other institutions, the switch to online meetings perhaps facilitated even better engagement in our discussions. Hence, there was no interruption to JCI activities.

The lockdown and social isolation that followed the onset of the pandemic significantly reduced personal interactions and the continuing board meetings were welcomed by many board members, as they provided a mechanism for sustained interaction and sharing information about the situation. The early virtual meetings inevitably included discussion of what was happening nationally and at our hospitals. This allowed us to share the situation in Baltimore as well as in other locations where our editors were based, including New York, Nashville, Boston, and Washington D.C. This shift occurred at the same time that the lockdown created isolation from colleagues and separation from our laboratories and scientific work. For many, the weekly meetings with colleagues on the JCI Editorial Board served as a lifeline for personal contact both scientifically and nonscientifically. The board meetings provided a weekly forum to discuss science and constituted a mechanism to "see" colleagues regularly, at least virtually (Figure 1), thus providing a bit of normalcy in our otherwise disrupted personal and professional lives.

The cessation of most work-related activities and the transition to working from home that occurred by the end of March 2020 was associated with a large increase in manuscript submissions at the JCI. In April 2020, the JCI received 497 manuscripts, representing a $66 \%$ increase in submissions over the average of 299 from the same month in the prior three years. Since the laboratories in many countries, including much of the United States, were closed by stay-at-home orders, this increase in submissions must have reflected a greater emphasis by investigators on writing up and submitting previously obtained research data. In this regard it is also possible that the cessation in scientifically related travel also freed up time for completion of manuscripts. The volume of manuscripts received by the JCI peaked in April to June 2020, and even though it has declined somewhat since then, it remained elevated in much of 2021 relative to the three years prior to 2020 (Figure 2).

The increase in submissions to the JCI occurred at the same time as there was a significant mobilization of editors and reviewers for COVID-19-related work. Some editors had to assume greater clinical responsibilities as our hospitals began to care for a large increase in COVID-19related hospital admissions. This was the case for several editors in the subspecialty of infectious diseases, who faced increased hospital responsibilities precisely at the same time when more papers dealing with COVID-19 were arriving at the JCI. To deal with this situation, editors with expertise in other fields in medicine stepped in to help. Thus, the pandemic 


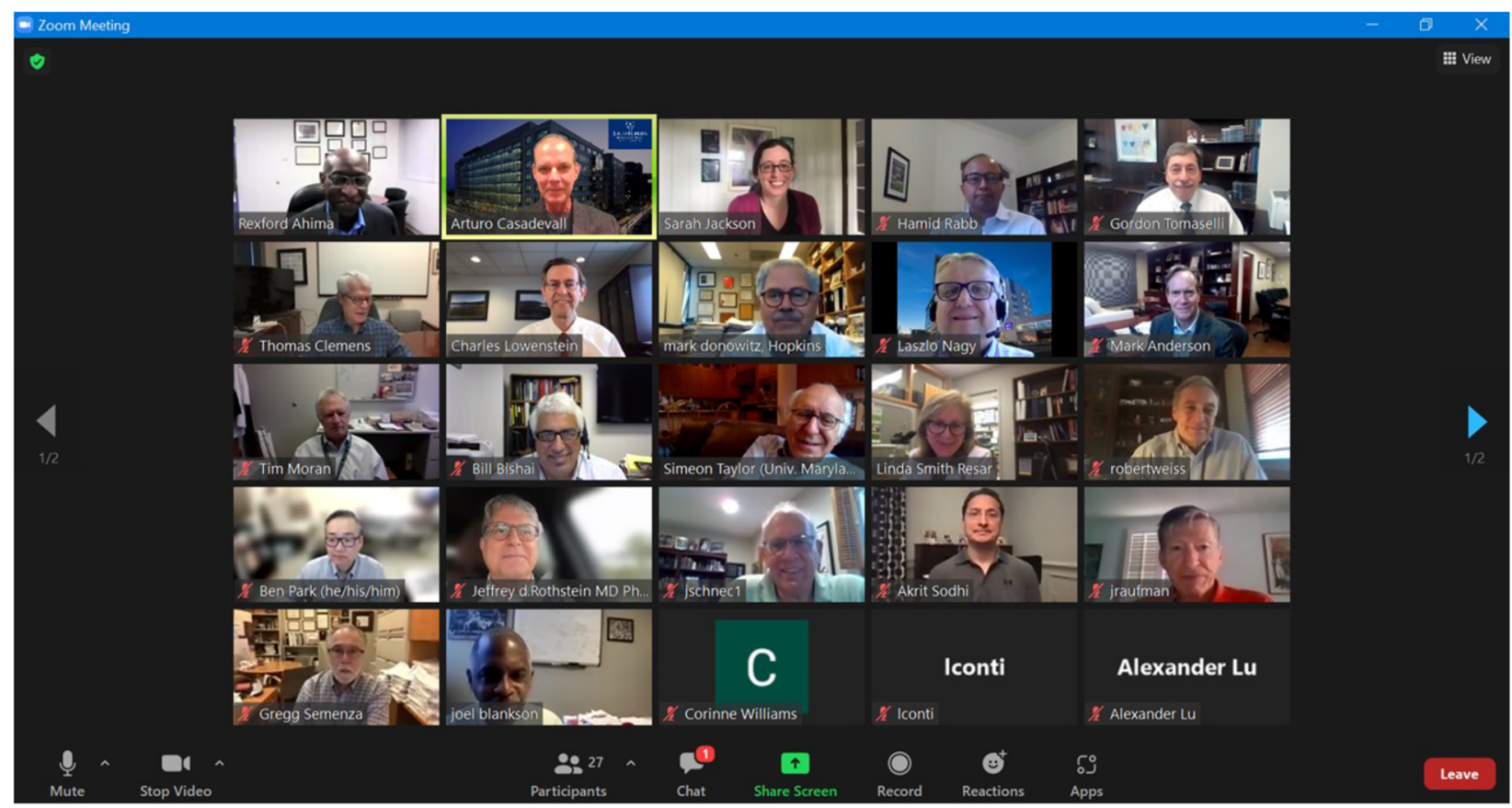

Figure 1. Computer screen shot of the JCI Editorial Board meeting on October 12, 2021.

forced many JCI editors to become more acquainted with virology, immunology, and public health disciplines, leaving the comfort zones of their own specialties. Adding to the difficulties, it became harder to find reviewers and many of the reviews received were shorter and less thorough than had been the norm. Hence, there were more manuscripts to deal with but fewer editorial and reviewer resources to handle them. Despite these stresses the JCI continued to function well, and we were able to maintain an average time to decision of under 30 days for papers sent for external review. Our external reviewers, particularly of COVID-19-related submissions, were heavily taxed with requests to review papers, but most appreciated the utmost importance of their work and the urgency with which their expertise was required. This allowed us to make fast decisions on COVID-19-related submissions, and we are truly indebted to the reviewers for their service.

\section{Pandemic response and contributions}

The JCI played a major role in the pandemic response by publishing two early papers on the potential of convalescent plasma (CP) therapy for COVID-19. One of us (AC) wrote an OpEd that was published in the Wall Street Journal on February 28, 2020, reminding the public and physicians that CP had a long history of therapeutic usefulness against a variety of infectious diseases (3). However, there was no good reference source to guide the preparation of investigational review board applications for the use of CP. Consequently, Drs. Casadevall and Pirofski wrote a Viewpoint for the JCI to provide the rationale, background material, and references needed to inform physicians considering this option (4). The Viewpoint was posted on the JCI website on March 13, 2020, and it was the first COVID-19-related publication in the journal. This paper was downloaded tens of thousands of times in the early weeks of the pandemic (and to date has received 377,260 views) and became a sort of manifesto for a grass roots movement to use CP that culminated when the FDA established the Expanded Access Program making CP available in the United States (5). However, it soon became clear that CP was a very complex therapy to deploy, requiring hematological, blood banking, infectious disease, and immunological expertise, and there was a need for a "how to" publication that brought those disciplines together. To meet this challenge, Evan Bloch recruited the necessary experts to write another piece on the topic that was published shortly thereafter (6). Subsequently, the JCI published the first detailed analysis of antibody content and function in CP showing that the intensity of the immune response was associated with the severity of the disease and that males had stronger responses than females (7). Early in the pandemic there was concern that antibody-based therapies would worsen disease through an effect known as antibody-dependent enhancement (8), but this concern was lessened when the JCI published a report that CP use in 5000 patients was as safe as plasma (9). With regard to CP efficacy, the JCI published the first double-blind placebo-controlled trial reporting that $\mathrm{CP}$ administration reduced mortality by about $50 \%$ (10).

By November 2021, the JCI had published 120 COVID-19-related papers. We highlight here selected contributions that we consider among the most important papers published during the pandemic. The first research article that the JCI published was "Clinical and immunological features of severe and moderate coronavirus disease 2019" (11), which went online on March 27, 2020, after submission on February 12, 2020. This article from Chinese investigators provided the first detailed descriptions of clinical and immunological characteristics of 


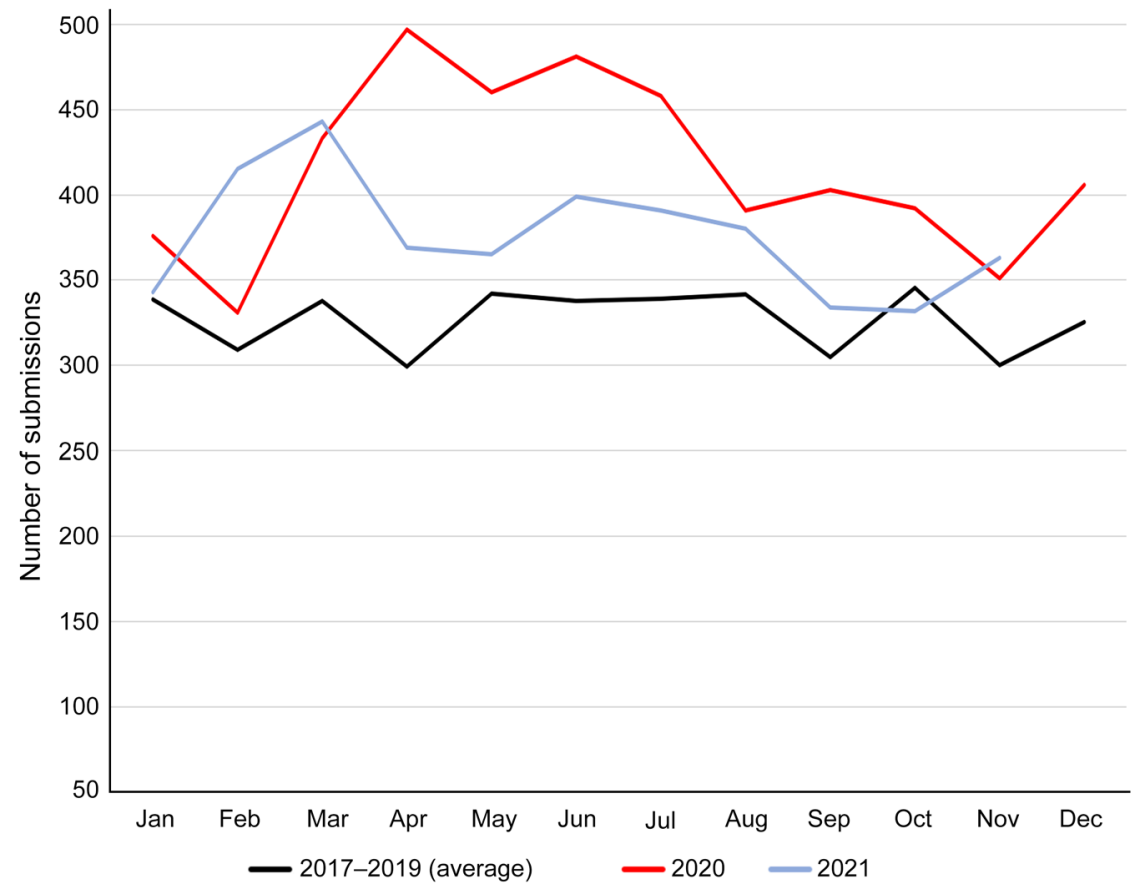

Figure 2. Number of manuscripts submitted to the $J C l$ during and immediately before the pandemic. Submissions from 2021 (January to October) and 2020 are compared with the average submissions during the period 2017 to 2019.

the disease and filled an early need for COVID-19 information. By the time of this writing, this paper and its accompanying Commentary had 3043 and 785 citations, respectively. The JCI published two of the foundational pediatric COVID-19 papers in 2020 describing multisystem inflammatory syndrome in children (MIS-C) (12, 13), and in 2021 reported a mechanism for the disease based on viral infection of the gut triggering increased systemic leakage of microbial antigens (14). On the topic of susceptibility to COVID-19, the JCI published articles reporting that prior recent infection with coronaviruses led to less severe disease (15), that age-related waning of $\mathrm{T}$ cell immunity was associated with worse disease (16), that $\mathrm{T}$ cell imprinting from prior coronavirus infections could impair the immune response (17), and that there was a lack of association of severe disease with interferon (IFN) gene polymorphisms (18) or IFN reactive antibodies (19). In the area of COVID-19 pathogenesis, the JCI published research demonstrating that nasal ciliated cells were sites for early infection (20), high interleukin 6 levels were associated with impaired cellular cytotoxic activity (21), the placenta could be affected by SARS-CoV-2 (22), phospholipase A2 (23) and complement (24) contributed to severe disease, and even in asymptomatic disease there were alterations in brain blood flow (25). With regard to vaccines, the JCI published articles showing that the mRNA vaccines were likely to confer protection to viral variants (26), vaccine-elicited antibody crossed the placenta to transfer immunity to neonates (27), and vaccination after COVID-19 elicited strong immune responses $(28,29)$.

\section{Pandemic reflections}

Considering JCI operations with the insight of the past one and a half years, one can argue that the pandemic changed everything, but everything remained pretty much the same. Day-to-day operations transitioned from office to home settings but the fundamental job of the journal, which is to handle the more than 3000 submissions received each year, identify top papers, arrange for peer review, and then publish those that meet the highest standards, remained the same (30). Nevertheless, when judging the quality and importance of submissions, the JCI board did take into consideration the medical emergency and the need for providing high-quality, peer-reviewed information when evaluating certain COVID-19 manuscripts. In normal times, the JCI board has a definite preference for scientific papers that report cause-and-effect studies, or to use the inexact parlance of our day, mechanistic over descriptive science $(31,32)$. However, as the world was overtaken by the pandemic, the board members recognized the tremendous need for basic information about a devastating new disease and many of our early publications that described the pathophysiology of COVID-19 lacked the type of mechanistic detail that is a hallmark of JCI papers. However, once foundational information on the pathophysiology, immune response, and epidemiology was available, the board increased its stringency and expectations for depth in COVID-19-related submissions. By the second year of the pandemic the board returned to its usual criteria of preferring papers that reported complete stories that explored cause-and-effect relationships while referring many observational, descriptive, and correlational COVID-19 studies to other more specialized journals.

In evaluating the novelty of manuscripts, the JCI board had to contend with the fact that much of the early information on COVID-19 epidemiology, pathogenesis, and therapy was made public through press releases and/or the posting of research papers prior to peer review onto preprint servers. In fact, many of the key early papers of the pandemic were discussed by the press months before they were published in peer-reviewed journals. Furthermore, the pace of discovery was at times so rapid that by the time a manuscript submitted to the $J C I$ was reviewed and discussed at board meetings, the information it contained was superseded by other studies or was common knowledge among physicians. This was the case for many papers where the time from submission to review took many weeks, given the pandemic-related delays in finding reviewers and editors. In this situation, the board took the position that there was a need to publish high-quality, peerreviewed information, and hence focused only on the excellence of the work submitted in making its decisions.

The JCI's experience during COVID-19 showed that journals have a critical role to play in pandemics by providing venues for 
responsible vetting of information that can impact the response to the calamity. In fact, this mission became even more important at a time when there was a loosening of the mechanisms by which medical information was transmitted to the public, including the frequent use of press releases to announce medical findings and the rise of preprints (33). During the COVID-19 crisis, the Editorial Board of the JCI experienced increased submissions, a need for timely release of information, and disruption to its operations as the lives of editors, reviewers, and journal staff were upended by changes in professional and personal responsibilities. These stresses on the system challenged us to consider what is possible and what is sustainable in scientific publishing, and reinforced our mandate to publish the very best science of clinical relevance. We are proud that the JCI functioned without interruption and delivered in its mission of publishing first-class medical research while providing editors with a medium for continued meaningful interactions during a time of social isolation and stress.

\section{Acknowledgments}

The title of this article is inspired by the 1985 novel Love in the Time of Cholera, by Nobel Prize-winning author Gabriel García Márquez. The authors wish to thank all of the JCI's authors, reviewers, board members, and journal staff for their tireless efforts and without whom none of these accomplishments would be possible.

\section{Arturo Casadevall, Sarah Jackson, Gregg L. Semenza, Gordon F. Tomaselli, and Rexford S. Ahima}

1. Holshue ML, et al. First case of 2019 novel coronavirus in the United States. N Engl J Med. 2020;382(10):929-936.

2. Howell JD. A history of the American Society for Clinical Investigation. J Clin Invest. 2009;119(4):682-697.
3. Casadevall A. How a boy's blood stopped an outbreak. Wall Street Journal. February 27, 2020 Accessed November 3, 2021. https://www.wsj. com/articles/how-a-boys-blood-stopped-anoutbreak-11582847330.

4. Casadevall A, Pirofski LA. The convalescent sera option for containing COVID-19. JClin Invest. 2020;130(4):1545-1548.

5. Senefeld JW, et al. Program and patient characteristics for the United States Expanded Access Program to COVID-19 convalescent plasma [preprint]. https://doi.org/10.1101/2021.04.08. 21255115. Posted on medRxiv April 10, 2021

6. Bloch EM, et al. Deployment of convalescent plasma for the prevention and treatment of COVID-19. J Clin Invest. 2020;130(6):2757-2765.

7. Klein SL, et al. Sex, age, and hospitalization drive antibody responses in a COVID-19 convalescent plasma donor population. J Clin Invest. 2020;130(11):6141-6150.

8. Arvin AM, et al. A perspective on potential antibody-dependent enhancement of SARS-CoV-2. Nature. 2020;584(7821):353-363.

9. Joyner MJ, et al. Early safety indicators of COVID-19 convalescent plasma in 5000 patients. J Clin Invest. 2020;130(9):4791-4797.

10. O'Donnell MR, et al. A randomized double-blind controlled trial of convalescent plasma in adults with severe COVID-19. J Clin Invest. 2021;131(13):e150646.

11. Chen G, et al. Clinical and immunological features of severe and moderate coronavirus disease 2019. J Clin Invest. 2020;130(5):2620-2629.

12. Diorio C, et al. Multisystem inflammatory syndrome in children and COVID-19 are distinct presentations of SARS-CoV-2. JClin Invest. 2020;130(11):5967-5975

13. Lee PY, et al. Distinct clinical and immunological features of SARS-CoV-2-induced multisystem inflammatory syndrome in children. J Clin Invest. 2020;130(11):5942-5950.

14. Yonker LM, et al. Multisystem inflammatory syndrome in children is driven by zonulindependent loss of gut mucosal barrier. J Clin Invest. 2021;131(14):149633

15. Sagar M, et al. Recent endemic coronavirus infection is associated with less-severe COVID19. J Clin Invest. 2021;131(1):143380.

16. Sattler A, et al. SARS-CoV-2-specific T cell responses and correlations with COVID-19 patient predisposition. J Clin Invest. 2020;130(12):6477-6489.

17. Dykema AG, et al. Functional characterization of CD4+ T cell receptors crossreactive for SARSCoV-2 and endemic coronaviruses. J Clin Invest. 2021;131(10):146922.
18. Povysil G, et al. Rare loss-of-function variants in type I IFN immunity genes are not associated with severe COVID-19. J Clin Invest. 2021;131(14):147834.

19. Meisel C, et al. Mild COVID-19 despite autoantibodies against type I IFNs in autoimmune polyendocrine syndrome type 1. J Clin Invest. 2021;131(14):150867.

20. Ahn JH, et al. Nasal iliated cells are primary targets for SARS-CoV-2 replication in the early stage of COVID-19. J Clin Invest. 2021;131(13):148517.

21. Mazzoni A, et al. Impaired immune cell cytotoxicity in severe COVID-19 is IL-6 dependent. JClin Invest. 2020;130(9):4694-4703.

22. Hosier H, et al. SARS-CoV-2 infection of the placenta. JClin Invest. 2020;130(9):4947-4953.

23. Snider JM, et al. Group IIA secreted phospholipase A2 is associated with the pathobiology leading to COVID-19 mortality. J Clin Invest. 2021;131(19):e149236.

24. Skendros P, et al. Complement and tissue factor-enriched neutrophil extracellular traps are key drivers in COVID-19 immunothrombosis. J Clin Invest. 2020;130(11):6151-6157.

25. Qin Y, et al. Long-term microstructure and cerebral blood flow changes in patients recovered from COVID-19 without neurological manifestations. JClin Invest. 2021;131(8):147329.

26. Woldemeskel BA, et al. SARS-CoV-2 mRNA vaccines induce broad $\mathrm{CD} 4+\mathrm{T}$ cell responses that recognize SARS-CoV-2 variants and $\mathrm{HCoV}$ NL63. JClin Invest. 2021;131(10):149335.

27. Beharier O, et al. Efficient maternal to neonatal transfer of antibodies against SARS-CoV-2 and BNT162b2 mRNA COVID-19 vaccine. J Clin Invest. 2021;131(13): 150319.

28. Mazzoni A, et al. First-dose mRNA vaccination is sufficient to reactivate immunological memory to SARS-CoV-2 in subjects who have recovered from COVID-19. J Clin Invest. 2021;131(12):e149150

29. Levi R, et al. One dose of SARS-CoV-2 vaccine exponentially increases antibodies in individuals who have recovered from symptomatic COVID-19. J Clin Invest. 2021;131(12):149154.

30. Ahima RS, et al. Changing the editorial process at JCI and JCI Insight in response to the COVID19 pandemic. J Clin Invest. 2020;130(5):2147.

31. Casadevall A, Fang FC. Descriptive science. Infect Immun . 2008;76(9):3835-3836.

32. Casadevall A, Fang FC. Mechanistic science. Infect Immun. 2009;77(9):3517-3519.

33. Saitz R, Schwitzer G. Communicating science in the time of a pandemic. JAMA. 2020;324(5):443-444. 